\title{
EFECTO DE LAS TÉCNICAS DE MEJORA ECOHIDROLÓGICA DEL PASTIZAL SOBRE EL RENDIMIENTO HÍDRICO DE LA MICROCUENCA ALTO ANDINA URPAY
}

\author{
EFFECT OF GRASSLAND ECOHYDROLOGICAL IMPROVEMENT \\ TECHNIQUES ON THE WATER PERFORMANCE OF HIGH ANDEAN MICRO \\ WATERSHED URPAY
}

\author{
Luis Oscanoa ${ }^{1}$ y Enrique Flores ${ }^{2}$
}

\begin{abstract}
Resumen
El presente trabajo fue realizado en el ecosistema de montañas en deterioro de la comunidad campesina Cordillera Blanca, Huaraz - Perú. El objetivo fue desarrollar una propuesta de estudio basada en la aplicación integrada de los índices de la condición del pastizal (C), humedad del suelo $(\mathrm{H})$, caracterización ecohidrológica y mejora del rendimiento hídrico (RH) con "surcos" y "hoyos". La C fue estudiada con el método Parker (1951), la $\mathrm{H}$ con el diseño experimental completamente al azar y arreglo factorial (3 técnicas x 6 épocas por parámetro de estudio) y el RH con el aplicativo HEC-HMS. La investigación duró dos años, etapa en la que se estudiaron la mejora de la $\mathrm{C} \mathrm{y} \mathrm{H}$, atributos base para el mapeo ecohidrológico (Vulnerabilidad, conservación, función hidrológica, retención de agua) y el RH. La C muy pobre no mostró cambios pero sí la $\mathrm{H}(\mathrm{P}=0.000<0.05)$, siendo los "surcos" superior (14.66\%) a "hoyos" (12.95\%) y control (10.95\%); el mapeo ecohidrológico del territorio (2 449 ha) identificó aptitudes de calidad media (49\%), alta (29\%) y pobre (22\%); y los "surcos" perdieron menos caudal de agua $\left(1.17 \mathrm{~m}^{3} \cdot \mathrm{s}^{-1}\right)$ que "hoyos" $\left(1.19 \mathrm{~m}^{3} \cdot \mathrm{s}^{-1}\right)$ y el control $\left(1.20 \mathrm{~m}^{3} \cdot \mathrm{s}^{-1}\right)$. Se concluye que la caracterización ecohidrológica proporcionó adecuados indicadores para el diseño del plan de conservación, restauración y monitoreo de la microcuenca; los índices C y H son muy confiables y los "surcos" y "hoyos" son técnicas efectivas para la restauración de la función ecohidrológica del pastizal.
\end{abstract}

Palabras clave: pastos naturales, microcuenca, ecohidrología, rendimiento hídrico.

\begin{abstract}
The present work was performed in a deteriorated mountain ecosystem in a peasant community from the Cordillera Blanca, Huaraz - Peru. The objective of the experiment was to develop a study proposal based on the integrated application of pasture condition indexes $(\mathrm{C})$, soil humidity $(\mathrm{H})$, ecohydrological characterization (EH) and improved water performance (RH) using "furrows" and "pits". The C was studied with the Parker method (Parker, 1951), the H with a completely randomized experimental design with a factorial arrangement $(3 \times 6$ times techniques by study parameter) and the RH with the HEC-HMS applicative. The study lasted two years, period in which the improvement of $\mathrm{C}$ and $\mathrm{H}$ were studied, which constituted base attributes for the ecohydrological mapping (Vulnerability, conservation, hydrological function, water retention) and RH. A very poor $\mathrm{C}$ did not show changes but $\mathrm{H}$ did $(\mathrm{P}=0.000<0.05)$. "Furrows" were higher $(14.66 \%)$ than "pits" $(12.95 \%)$ and control (10.95\%). Ecohydrological mapping of the area (2 449 ha) identified medium (49\%), high (29\%) and poor (22\%) qualities. "Furrows" lost less water flow $\left(1.17 \mathrm{~m}^{3} . \mathrm{s}^{-1}\right)$ than "pits" $\left(1.19 \mathrm{~m}^{3} \cdot \mathrm{s}^{-1}\right)$ and control $\left(1.20 \mathrm{~m}^{3} \cdot \mathrm{s}^{-1}\right)$. It is concluded that ecohydrological characterization provided appropriate indicators for designing conservation plans, restoration and monitoring of micro watersheds; $\mathrm{C}$ and $\mathrm{H}$ indexes are of high confidence. "Furrows" and "pits" are effective techniques for the restoration of the water function of the pasture.
\end{abstract}

Key words: grassland, micro watersheds, ecohydrological, water performance.

\section{Introducción}

En cuencas hidrográficas, se han ensayado modelos hidrológicos para estimar la descarga de agua y erosión del suelo. Los parámetros utilizados para el análisis del ordenamiento territorial fueron: biomasa de cultivos
(Pieri et al., 2007), precipitación-escorrentía (Amaya et al., 2009), erosión del suelo (Nearing et al., 2011), cobertura vegetal (Di Luzio et al., 2002) y uso de la tierra (Albhaisi et al., 2013). En este sentido, Nearing et al. (2011) desarrollaron el Range Hidrologic Erotion 
Model (RHEM) con el fin de modelar la erosión del pastizal basado en informaciones de tierras de cultivo (escorrentía y erosión) ellos consideraron de que las tasas de pérdida de suelo son uno de los pocos indicadores para evaluar la salud del pastizal y la eficiencia práctica de su conservación.

Uno de los problemas preponderantes en la gestión hídrica de ecosistemas de montañas es el sobrepastoreo del pastizal. En esta, las especies vegetales del pastizal afectan diferenciadamente el desarrollo de los parámetros composición florística, biomasa aérea y humedad del suelo del ecosistema pastizal y las correlaciones entre estas son cambiantes con la época del año (Eviner, 2004); de la misma forma, el estado fenológico de las plantas varía con la especie, el suelo y el clima (Tovar \& Oscanoa, 2002). Por problemas de variabilidad del clima, el modelamiento requiere considerar un enfoque integral para el diseño de planes de control de los servicios ecosistémicos porque la duración y la intensidad de pastoreo incrementa la escorrentía y erosión del suelo, amenazas constantes para la desertificación y la condición de los pastizales (Zhang et al., 2012; Yalew, 2014).

Desde el punto de vista de la ciencia del pastizal, Florez \& Bryant (1989) definieron como "condición" al diagnóstico de los cambios que ocurren con respecto a la comunidad clímax (situación de máxima relación trófica e equilibrio del ecosistema). Pyke et al. (2002) recomendaron incorporar para estudios de suelos, cubiertos de pastos naturales el enfoque ecológico, involucrando para ello atributos de estabilidad del sitio, función hidrológica e integridad biótica de la comunidad vegetal que anteriores metodologías no lo consideraron. Y desde la ciencia del suelo se propone que en los próximos estudios de la condición ecológica del pastizal se integre al modelo de evaluación el factor humedad del suelo que muy pocas veces se tuvieron en cuenta (Eekeren et al., 2010; Oscanoa \& Flores, 2016). Snymana \& Preez (2005) encontraron que los cambios en los niveles de humedad del suelo varían en concordancia al grado de degradación del suelo y siendo la lluvia la única fuente de recarga de agua en el pastizal estas muestran influencia significativa en la cantidad de humedad del suelo (Salve \& Allen-Diaz, 2001).

El objetivo de este trabajo fue desarrollar una propuesta basada en la aplicación integrada de los principales atributos de la ciencia del pastizal (condición - C) y suelo (humedad - H), caracterización ecohidrológica y simulación de mejora del rendimiento hídrico (RH) de la microcuenca con las técnicas de "hoyos" y "surcos" de infiltración. Las preguntas de investigación fueron ¿cuál es el nivel de influencia de los atributos de la condición del pastizal y humedad del

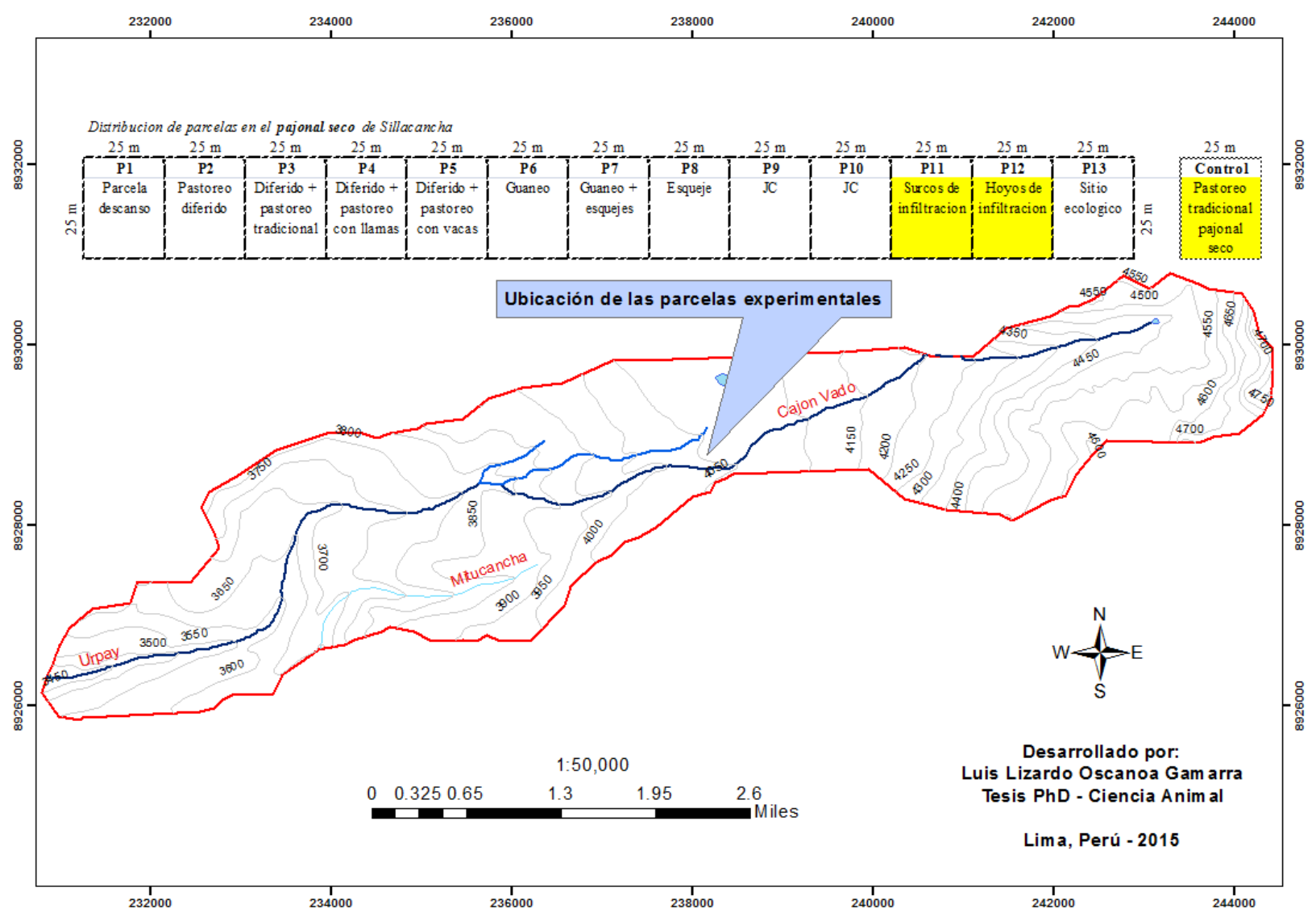

Figura 1. Ubicación de las parcelas experimentales en la microcuenca Urpay. 


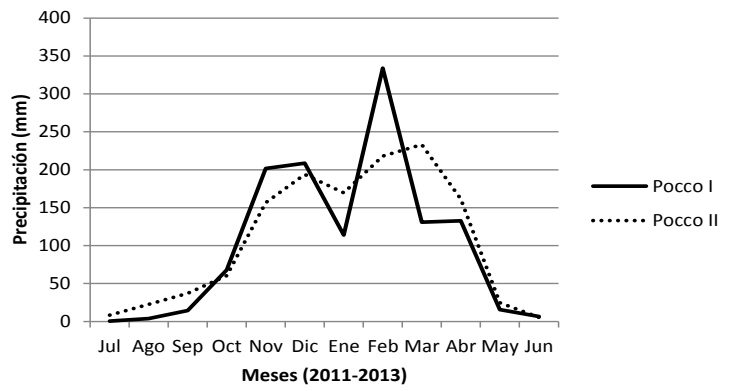

Figura 2. Precipitación en la estación pluviométrica Pocco I y II (Gil, 2014).

suelo?, ¿son los submodelos de vulnerabilidad, conservación, función hidrológica y retención del agua adecuados para el estudio ecohidrológico del pastizal?, ¿cuál es el nivel de mejora del rendimiento hídrico con las técnicas "hoyos" y "surcos"?

\section{Materiales y métodos}

\section{Diseño del estudio y participantes}

Llevamos a cabo este trabajo en pastos naturales alto andinos (4 $100 \mathrm{msnm}$ ) entre mayo 2011 y diciembre 2014 en terrenos de la comunidad campesina Cordillera Blanca, Sector Canrey Chico, Provincia de Recuay, Huaraz, Perú (Figura 1). La delimitación de la subcuenca Urpay se realizó participativamente con ganaderos de la comunidad y el apoyo de imágenes satelitales Landsat con sensor TM del año 2005 aportada por el Ministerio de Educación del Perú (MINEDU). Holdridge (1987) clasificó a esta zona como Páramo muy Húmedo-Subalpino Tropical (Pmh$\mathrm{SaT}$ ) con temperatura promedio de $12{ }^{\circ} \mathrm{C}$, precipitación promedio de $700 \mathrm{~mm}$ por año y suelo franco arenoso con tipo de pastizal Calamagrostis macrophylla y Stipa brachyphylla de condición muy pobre.

\section{Población del estudio}

La unidad de análisis del comportamiento hídrico del pastizal fue la microcuenca Urpay, ubicada a una altitud de 3450 - 4800 msnm y con punto de descarga tributaria en la coordenada UTM 230800 y 8926312 (Figura 1). En la cabecera del territorio nace el rio Urpay generado por la caída de lluvias. En la Figura 2 se presenta las precipitaciones registradas por las estaciones pluviométricas Pocco I y Pocco II, implementadas durante el periodo de estudio. El caudal base fue sostenido en el tiempo por pequeñas lagunas, puquiales, bofedales y filtraciones que proveyeron agua al rio, las que finalmente como tributario desembocaron en el rio Santa. La microcuenca fue considerada representativa del sistema de cabeceras de cuenca de la cordillera blanca y proporcionó información confiable para la simulación del comportamiento hidrológico del ecosistema pastizal alto andino. Es importante este trabajo debido a que la influencia de los atributos del estado ecohidrológico sobre la función productiva de la microcuenca se encuentra inmersa dentro un sistema dinámico de alta complejidad.

Tratamientos Experimentales

Se ensayaron 18 tratamientos producto de la combinación de tres técnicas y seis épocas de muestreo. El monitoreo detallado de los tratamientos se realizó bajo el modelo sistémico aleatorio en parcelas ("surcos", "hoyos" y uso comunal) en las épocas de fin de lluvias (may-11), plena seca (ago-11), inicio de lluvias (oct-11), plena lluvia (feb-13) y plena seca (set13) consideradas estaciones claves para el manejo ganadero. Estos tratamientos fueron estudiados en parcelas cercadas $25 \times 25 \mathrm{~m}$, donde los surcos $(15 \mathrm{~cm}$ de ancho por $10 \mathrm{~cm}$ de profundidad) fueron construidos a intervalos de dos metros y los hoyos $(7.5 \mathrm{~cm}$ diámetro por $10 \mathrm{~cm}$ de profundidad) a intervalos de un metro, en referencia al área de pastoreo libre comunal considerada como testigo (Figura 1).

Parámetros Evaluados

La condición del pastizal de las parcelas se evaluó al inicio y final del experimento con la metodología propuesta por Oscanoa \& Flores (2016) a través de los índices de especies deseables (D en \%), índice forrajero (IF $=\%$ de especies deseables más poco deseables), índice de suelo desnudo más pavimento de erosión más roca (BRP en \%) y el índice de vigor (V en \%) de las especies vegetales claves (Parker, 1951). La integración matemática de los índices parciales de condición se realizó con la Fórmula:

Puntaje $=0.5 \mathrm{D}+0.2 \mathrm{IF}+0.2 \mathrm{COB}+0.1 \mathrm{~V}$,

Donde:

Índice de cobertura $(\mathrm{COB})=100-\mathrm{BRP}$

El puntaje total se comparó con la escala puntaje condición (Tabla 1) propuesta por Florez et al. (1991).

Las muestras de suelo para el análisis de la humedad del suelo fueron obtenidos con cilindros de muestreo de $7.62 \mathrm{~cm}$ de altura y $7.62 \mathrm{~cm}$ de diámetro a 10 y $20 \mathrm{~cm}$ de profundidad del suelo. Con una comba se introdujo

Tabla 1. Escala puntaje condición y carga optima por especie animal.

\begin{tabular}{|c|c|c|c|c|c|c|}
\hline \multirow{2}{*}{$\begin{array}{l}\text { Condición del } \\
\text { pastizal }\end{array}$} & \multirow[t]{2}{*}{ Puntaje (\%) } & \multicolumn{5}{|c|}{ Carga animal en pastoreo excluyente (ha/año) } \\
\hline & & Ovinos & Alpacas & Llamas & Vacunos & Vicuñas \\
\hline Excelente & $81-100$ & 4.00 & 2.70 & 1.80 & 1.00 & 4.44 \\
\hline Bueno & $61-80$ & 3.00 & 2.00 & 1.30 & 0.75 & 3.33 \\
\hline Regular & $41-60$ & 1.50 & 1.00 & 0.70 & 0.38 & 1.65 \\
\hline Pobre & $21-40$ & 0.50 & 0.33 & 0.20 & 0.13 & 0.55 \\
\hline Muy pobre & $1-20$ & 0.25 & 0.10 & 0.10 & 0.07 & 0.28 \\
\hline
\end{tabular}

Fuente: Florez et al. (1991). 
el cilindro en el suelo, se uniformizó la superficie de los extremos del cilindro con cuchillo plano, se sellaron con papel de hornear y las muestras codificadas fueron trasladadas al laboratorio (Oscanoa \& Flores, 2016). En el laboratorio, las muestras frescas fueron pesadas, oreadas y secadas en estufa a $105^{\circ} \mathrm{C}$ por 24 horas y luego las muestras secas fueron pesadas en balanza de precisión y los resultados expresados en porcentaje (Blake et al., 1965).

La caracterización ecohidrológica se realizó con el objeto de integrar los parámetros ecológicos e hidrológicos del pastizal para la restauración de las funciones ambientales del ecosistema; en razón del cual se generó los submodelos de vulnerabilidad, estado de conservación, función hidrológica y capacidad de retención de agua por el suelo (Pyke et al., 2002; Oscanoa, 2016); el primero evaluó el daño provocado por el hombre, en razón del cual se consideraron los indicadores de fisionomía vegetal, pendiente y precipitación; el segundo submodelo midió el efecto del sobre pastoreo sobre el estado de la comunidad vegetal, a través de la condición del pastizal, modalidad de tenencia de tierras y práctica local de conservación de suelos; en el tercer submodelo se analizaron el efecto del comportamiento biofísico del agua de lluvias sobre la producción de biomasa, humedad del suelo, producción de mantillo y temperatura del suelo (Oscanoa \& Flores, 2016); y en el último submodelo se estudiaron la capacidad de retener el agua de lluvias en contra de la fuerza de gravedad a través de la erosión del suelo, tasa de infiltración y análisis de textura del suelo.

El modelamiento ecohidrológico de la subcuenca se realizó con el software ENVI 5.0 y ArcGis 10.0; con el primero se hizo la fotointerpretación del área de estudio y con el segundo el desarrollo de los mapas temáticos y el modelamiento final. Se procesó las características ecohidrológicas de la subcuenca tomando como indicadores el ponderado de ponderados de los submodelos temáticos de vulnerabilidad, conservación, función hidrológica y retención del agua. El proceso determinó las características hídricas de las unidades zonificadas que luego fueron sometidos a simulación de mejora de las áreas con potencial ecohidrológico de la subcuenca.

El grado de influencia de las prácticas de conservación de suelos ("hoyos", "surcos") sobre la variable caudal fueron estimados con el software HECHMS; de modo que los atributos fueron analizados detalladamente con la finalidad de caracterizar realísticamente la tipología ecohidrológica y sus implicancias sobre el rendimiento hídrico de la microcuenca. Los parámetros considerados fueron: (1) datos de la superficie, tasa de pérdida determinado con el método curva número (Balvanshi \& Tiwari, 2014), conversión de lluvia en escurrimiento con el método del Unit Hydrography (SCS) y el flujo base constante fue el valor más bajo de la época seca registrados por los vertederos de la estación Pocco (Gil, 2014), (2) la curva número ponderada fue estimada con el ArcGis 10.0, la impermeabilidad considerada fue del $5 \%$ y el tiempo de concentración (Tc) fue calculado con la ecuación Kirpich (Vélez \& Botero, 2011; Taghvaye et al., 2017):

$\mathrm{Tc}=0.000323(0.77 \mathrm{~L} / 0.385 \mathrm{~S})$

Dónde:

L es la longitud del cauce, $\mathrm{S}$ es la semisuma de la cota máxima y mínima dividido entre $\mathbf{L}$. Y para el parámetro tiempo de retardo el valor fue considerado como el $60 \%$ del tiempo de concentración.

Diseño Experimental

Se empleó el Diseño Completamente al Azar con arreglo factorial con dos factores A (dos técnicas de mejora y testigo) y B (seis meses de muestreo), donde los tratamientos considerados fueron la interacción de las técnicas con los meses de muestreo, cuyas combinaciones hicieron un total de 18 tratamientos. El Diseño Factorial fue no balanceado (desigual cantidad de observaciones por casilla) y fue completo, ya que se realizaron todas las posibles combinaciones entre los valores de las variables (Montgomery, 1991). Los promedios de los parámetros estudiados fueron comparados con el test de comparaciones múltiples Duncan $(\mathrm{p}<0.05)$.

Modelo Estadístico:

$Y i j k=\mu+\alpha i+\tau j+\alpha \tau i j+e i j k$

$\mathrm{i}=1,2,3 ; \mathrm{j}=1,2,3,4,5,6 ; \mathrm{k}=1,2 \ldots ., \mathrm{nij}$

Donde:

Yijk es la respuesta obtenida (rendimiento de parámetros hidrológicos del pastizal) en la unidad experimental correspondiente a la k-ésima repetición a la cual se le aplicó el i-ésimo nivel del factor A, con el j-ésimo nivel del factor B. $\mu$ es el efecto de la media general. $\alpha \mathrm{i}$ es el efecto del i-ésimo nivel del factor técnica de mejora (A). $\tau$ j es el efecto del j-ésimo nivel del factor época de muestreo (B). $\alpha \tau i j$ es el efecto de la interacción entre el i-ésimo nivel del factor A con el jésimo nivel del factor B. eijk es la variación del error asociado con las ijk unidades.

\section{Resultados y discusión \\ Humedad del suelo y condición del pastizal}

En dos años de estudio la condición del pastizal muy pobre para ovinos, vacunos y vicuñas no lograron mejora con ninguna de las técnicas aplicadas ("hoyos" y "surcos"); en cambio la humedad del suelo de "surcos" mostró superioridad significativa en los factores técnica, mes y su interacción $\left(\mathrm{R}^{2}=0.812\right.$; $\mathrm{p}<0.05$ ). Al comparar el factor mes (noviembre 2012 y febrero 2013) con los otros meses estudiados, estas mostraron diferencias estadísticas significativas en todas sus combinaciones (Tabla 2). 


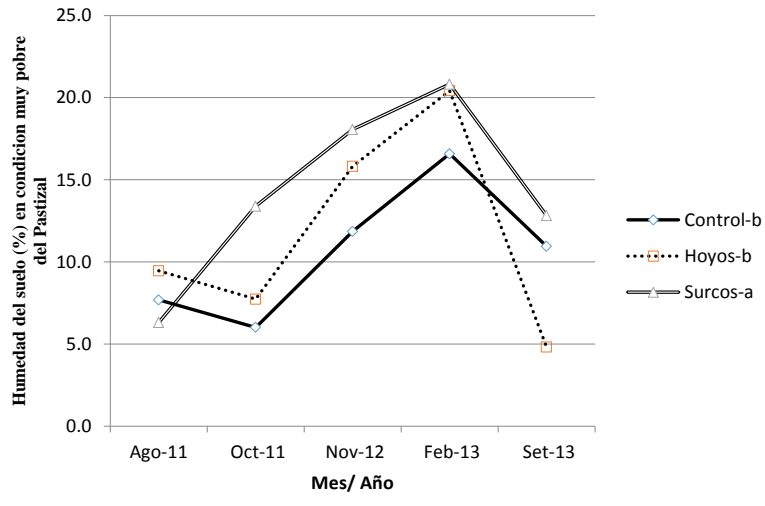

Figura 3. Humedad del suelo del pastizal muy pobre mejorado con "Hoyos" y "Surcos".

Estos datos sugieren que los atributos que evalúan el pastizal en deterioro, basado en la composición florística, están sujeto a una lenta recuperación del vigor de las plantas de importancia ganadera al aplicarse técnicas de mejora; sin embargo, el factor humedad del suelo se muestra más sensible a cambios que ocurren en el suelo; en ese sentido, los "surcos" mostraron superioridad sostenida en todos los meses estudiados y alcanzó el pico más alto durante el mes de febrero 2013 (Figura 3). Los resultados obtenidos expresan que los atributos ensayados son de alta complementariedad para la evaluación de la biomasa aérea del pastizal.

Deutsch et al. (2010) observaron que la humedad del suelo bajo sequía severa limita la producción de los pastizales templados-fríos, los cambios en los niveles de humedad del suelo variaron en concordancia al grado de degradación del suelo (Snymana \& Preez,
2005), siendo la lluvia la única fuente de recarga de agua; la textura, cubierta vegetal, y elevación influyen en el contenido de humedad del suelo (Salve \& AllenDiaz, 2001). La dinámica de la humedad del suelo mostraron tendencias similares en los perfiles del suelo de bosques y del pastizal con secado más intenso en el bosque durante la temporada de crecimiento, posiblemente por la mayor utilización de agua en las capas por los árboles (Hagyó et al., 2006). De manera que la función de los tipos de vegetación es afectada por la distribución de agua disponible en el suelo y la composición florística del pastizal (Breshears \& Barnes, 1999).

En los "hoyos", al final del experimento, hay un declive pronunciado del contenido de humedad del suelo, el cual podría deberse a que estas han perdido su función plena debido a que los hoyos se han rellenado nuevamente de suelo; este efecto es similar a la investigación comunicado por Al-Seekh et al. (2009) quienes observaron que la profundidad del suelo, precipitación y pisoteo del ganado son los principales factores para la pobre productividad de los pastizales. Zhang et al. (2010) determinaron que las tasas basales de respiración y temperatura varían con la gradiente de humedad, siendo la temperatura del suelo y contenido de agua los factores ambientales claves para la variación en la respiración del suelo; por lo que el manejo de los niveles de la humedad del suelo son parámetros importantes para el rendimiento de la función hidrológica del pastizal. En general, los resultados demostraron que las técnicas de mejora de suelos influenciaron en el incremento de la humedad del suelo y sustenta la confianza para la recuperación

Tabla 2. Estadísticos de la humedad del suelo de condición muy pobre del pastizal.

\begin{tabular}{|c|c|c|c|c|c|c|c|c|c|}
\hline \multirow[t]{2}{*}{ Técnicas } & \multirow[t]{2}{*}{ MES } & \multicolumn{2}{|c|}{ Humedad del Suelo } & \multicolumn{3}{|c|}{ Humedad del Suelo (ANOVA) } & \multicolumn{3}{|c|}{$\begin{array}{c}\text { Condición del Pastizal } \\
\text { Puntaje }(\%)\end{array}$} \\
\hline & & $\%$ & $\begin{array}{l}\text { Desviación } \\
\text { estándar }\end{array}$ & MES & $\begin{array}{c}\text { TECNICAS * } \\
\text { MES }\end{array}$ & DUNCAN & Ovinos & Vacunos & Vicuñas \\
\hline \multirow{5}{*}{$\begin{array}{l}\bar{\Xi} \\
\stackrel{\Xi}{0} \\
\dot{0}\end{array}$} & ago-11 & 7.7 & 4.49691 & & & \multirow{5}{*}{$\mathrm{b}$} & \multirow{3}{*}{12.0} & \multirow{3}{*}{12.0} & \multirow[b]{2}{*}{10.0} \\
\hline & oct- 11 & 6.0 & 1.35559 & & & & & & \\
\hline & nov-12 & 11.9 & 1.10589 & & & & & & \multirow{3}{*}{9.0} \\
\hline & set-13 & 11.0 & 1.32714 & & & & \multirow[t]{2}{*}{12.5} & \multirow[t]{2}{*}{12.5} & \\
\hline & Total ponderado & 10.9 & 4.61795 & & & & & & \\
\hline \multirow{6}{*}{$\begin{array}{l}\stackrel{0}{0} \\
\stackrel{0}{0} \\
\text { Th }\end{array}$} & ago-11 & 9.5 & 4.33268 & & & \multirow{6}{*}{$\mathrm{b}$} & \multirow{4}{*}{16.4} & \multirow{4}{*}{14.9} & \multirow{4}{*}{18.4} \\
\hline & oct-11 & 7.7 & 2.43004 & & & & & & \\
\hline & nov-12 & 15.8 & 2.26208 & & & & & & \\
\hline & feb-13 & 20.4 & 2.10879 & & & & & & \\
\hline & set-13 & 4.8 & 0.50619 & & & & \multirow[t]{3}{*}{16.6} & \multirow[t]{3}{*}{16.1} & \multirow[t]{3}{*}{17.1} \\
\hline & Total ponderado & 12.6 & 6.27149 & & & & & & \\
\hline \multirow{7}{*}{ 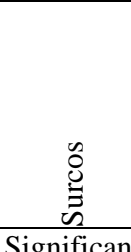 } & ago-11 & 6.3 & 0.86504 & & & & & & \\
\hline & oct-11 & 13.4 & 5.23276 & & & \multirow{5}{*}{$\mathrm{a}$} & \multirow[t]{3}{*}{12.5} & \multirow[t]{3}{*}{12.5} & \multirow[t]{2}{*}{12.5} \\
\hline & nov-12 & 18.1 & 2.62259 & & & & & & \\
\hline & feb-13 & 20.8 & 2.87176 & & & & & & \multirow{4}{*}{10.0} \\
\hline & set-13 & 12.8 & 1.28142 & & & & \multirow[t]{3}{*}{12.5} & \multirow[t]{3}{*}{12.5} & \\
\hline & Total ponderado & 14.7 & 5.64330 & & & & & & \\
\hline & $a(p<0.05)$ & & & 0.000 & 0.001 & 0.05 & & & \\
\hline
\end{tabular}

$\mathrm{R}^{\wedge} 2=0.812$. Condicion (Puntaje en $\%$ ) $=1-20 \Rightarrow$ Muy Pobre (MP).

Técnicas $=$ Técnicas de conservación de suelos. Letras diferentes $(\mathrm{a}, \mathrm{b})$ expresan diferencias estadísticas de medias. 
Tabla 3. Estado ecohidrológico de la subcuenca Urpay.

\begin{tabular}{|c|c|c|c|c|}
\hline & & le sub & & \\
\hline Vulnerabilidad (35\%) & Conservación (15\%) & Hidrología (30\%) & Retención de Agua (20\%) & Ponderado de ponderados \\
\hline
\end{tabular}

\begin{tabular}{|c|c|c|c|c|c|c|c|c|c|c|c|c|c|c|}
\hline 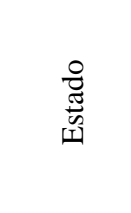 & 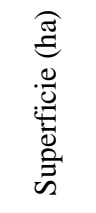 & 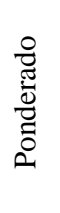 & 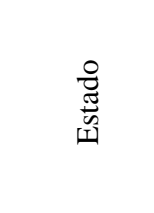 & 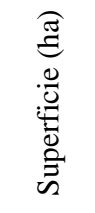 & $\begin{array}{l}\frac{0}{0} \\
\frac{\pi}{0} \\
\frac{0}{0} \\
0\end{array}$ & 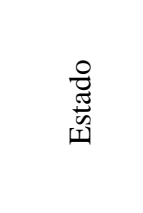 & 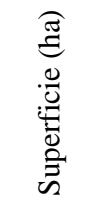 & 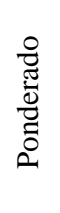 & 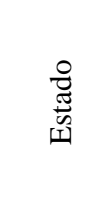 & 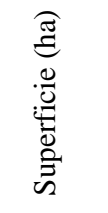 & $\begin{array}{l}\frac{8}{\pi} \\
\frac{\pi}{0} \\
\frac{0}{0} \\
0\end{array}$ & 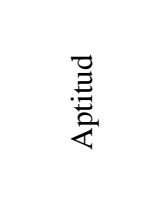 & 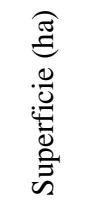 & 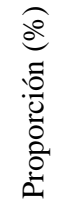 \\
\hline Bajo & 698 & 1 & Muy pobre & 1632 & 1 & Muy bajo & 424 & 1 & Bajo & 965 & 1 & Muy bajo & 117 & 5 \\
\hline Medio & 1219 & 2 & Pobre & 795 & 2 & Bajo & 998 & 2 & Medio & 1137 & 2 & Bajo & 427 & 17 \\
\hline Alto & 437 & 3 & Regulat & 77 & 3 & Medio & 638 & 3 & Alto & 399 & 3 & Medio & 1211 & 49 \\
\hline \multirow[t]{3}{*}{ Muy alto } & 124 & 4 & & & & Alto & 193 & 4 & & & & Alto & 700 & 29 \\
\hline & & & & & & Muy alto & 223 & 5 & & & & & & \\
\hline & 2478 & & & 2504 & & & 2476 & & & 2501 & & & 2455 & 100 \\
\hline
\end{tabular}

El valor porcentual (\%) se refiere al grado ponderado de influencia en los submodelos del estudio ecohidrológico de la microcuenca Urpay.

de las especies vegetales nativas de importancia ecohidrológica.

Modelo ecohidrológico de la microcuenca

El modelamiento determinó que la microcuenca Urpay ostenta sitios con vocación para la reserva de agua de lluvias. Proporcionalmente, de las 2445 ha del territorio, la extensión de bofedales y humedales representó el $29 \%$ de la extensión, las zonas de "aptitud media" fue de $49 \%$ y el $22 \%$ de la superficie restante mostraron signos de franco proceso de deterioro (Tabla 3). Superficialmente, estas significaron que las áreas se agruparon en media (1 $211 \mathrm{ha}$ ), baja (427 ha) y muy baja (117 ha), todas ellas con solo recarga de agua de lluvias y cubiertos por pastos naturales de puna y pajonales (Figura 4).

La ciencia ecohidrológica de montañas busca integrar los parámetros ecológicos e hidricos de los pastos naturales altoandinos con el propósito de mejorar las funciones ambientales dañados. En ese sentido dentro la tabla de valoracion del modelo ecohidrológico resaltan por nivel de importancia las temáticas de vulnerabilidad e hidrología del pastizal (Tabla 3). La primera expresa el mayor peso del efecto de vulnerabilidad para el ecosistema pastizal $(35 \%)$ ocacionados por la acción directa del hombre y la segunda el grado $(30 \%)$ de influencia del deterioro autoregulatorio de la caída de lluvias en la recarga natural del agua del rio Urpay (Figura 4).

Estos datos confirmaron que la aptitud ecohidrológica de la microcuenca se encontró íntimamente vinculada al tipo de uso de los pastos naturales, fisiografía y clima (inundación, friaje y sequia). Con respecto a los parámetros en estudio se encontró que el proceso ponderativo de los sub modelos fueron representativos, pues el modelo consolidado representó realísticamente la aptitud ecohidrológica de la microcuenca; de manera que las zonas calificadas como "medio" se muestran parcelas adecuados para la introducción de técnicas de mejora y la aptitud alta como área natural para la conservación hidrológica. A medida que se mejora el estudio geoespacial del ecosistema se fortalece la calidad de información para el desarrollo del plan de uso de los recursos naturales en armonía con el estado de las fuentes de agua.

Los gestores de recursos naturales requieren generar modelos explícitos de respuesta hidrológica a cambios de conductores climáticos en un escenario donde las condiciones de humedad futura son más difíciles de proyectar que la temperatura (Flint et al., 2013). El problema principal para el modelamiento es la poca disponibilidad de información hidroclimática (Amaya et al., 2009); por lo que el estado de pastoreo de terrenos tendría cuantitativamente ser evaluados por el estudio edafo-agrostológico (Florez \& Bryant, 1989) y la humedad del suelo (Snymana \& Preez, 2005; Salve

Tabla 4. Estadísticos descriptivos del rinde de descarga hídrica

\begin{tabular}{|c|c|c|c|c|}
\hline ÉPOCAS & $\begin{array}{c}\text { Prácticas de } \\
\text { conservación } \\
\text { de suelos }\end{array}$ & $\begin{array}{c}\text { Media } \\
\left(\mathbf{m}^{3} / \mathbf{s}\right)\end{array}$ & $\begin{array}{l}\text { Desviación } \\
\text { estandar }\end{array}$ & $\mathbf{N}^{\circ}$ \\
\hline \multirow{6}{*}{ Seca } & Actual & 0.31 & 0.29 & 123 \\
\hline & Hoyos & 0.31 & 0.29 & 123 \\
\hline & Surcos & 0.30 & 0.28 & 123 \\
\hline & Revegetación & 0.30 & 0.28 & 123 \\
\hline & Total ponderado & 0.30 & 0.28 & 492 \\
\hline & Actual & 0.98 & 1.16 & 91 \\
\hline \multirow{4}{*}{$\begin{array}{l}\text { Inicio de } \\
\text { lluvias }\end{array}$} & Hoyos & 0.98 & 1.16 & 91 \\
\hline & Surcos & 0.97 & 1.16 & 91 \\
\hline & Revegetación & 0.95 & 1.13 & 91 \\
\hline & Total ponderado & 0.97 & 1.15 & 364 \\
\hline \multirow{6}{*}{ Lluvias } & Actual & 2.29 & 1.47 & 91 \\
\hline & Hoyos & 2.29 & 1.47 & 91 \\
\hline & Surcos & 2.28 & 1.47 & 91 \\
\hline & Revegetación & 2.27 & 1.46 & 91 \\
\hline & Total ponderado & 2.28 & 1.46 & 364 \\
\hline & Actual & 1.67 & 1.45 & 61 \\
\hline \multirow{4}{*}{$\begin{array}{l}\text { Fin de } \\
\text { lluvias }\end{array}$} & Hoyos & 1.64 & 1.42 & 61 \\
\hline & Surcos & 1.57 & 1.37 & 61 \\
\hline & Revegetación & 1.43 & 1.28 & 61 \\
\hline & Total ponderado & 1.58 & 1.38 & 244 \\
\hline \multirow{5}{*}{ Total } & Actual & 1.20 & 1.36 & 366 \\
\hline & Hoyos & 1.19 & 1.36 & 366 \\
\hline & Surcos & 1.17 & 1.34 & 366 \\
\hline & Revegetación & 1.14 & 1.31 & 366 \\
\hline & Total ponderado & 1.17 & 1.34 & 1464 \\
\hline
\end{tabular}


\& Allen-Diaz, 2001); cualitativamente a través de los atributos de estabilidad del sitio, función hidrológica e integridad biótica de la comunidad vegetal (Pyke et al., 2002).

La existencia de factores de información por lo cual cada parámetro ecohidrológico pudo expresar su peso de influencia en el estudio de recarga de agua son índices dependientes del sistema hidrológico complejo que va más allá de la precipitación, tales como fuentes de deshielo del glaciar, filtraciones, lagunas, bofedales, y humedales. Los submodelos y modelos permitieron además ponderar adecuadamente el conocimiento local con propuestas metodológicas capaces de procesar integralmente las informaciones vinculadas a acuerdos comunales, testimonios de usuarios y percepciones locales.

Rendimiento hídrico de la microcuenca

La caracterización ecohidrológica de la microcuenca proporciono información razonable al modelo HEC-HMS para la estimación de descarga de agua de lluvias al implementarse las técnicas de mejora de suelos y calcular el efecto de mejora de la capacidad de retención de agua superficial de la microcuenca. En promedio la técnica "surcos" $\left(1.17 \mathrm{~m}^{3} . \mathrm{s}^{-1}\right)$ mostró un menor nivel de descarga en el punto final de recorrido del rio Urpay que los "hoyos" $\left(1.19 \mathrm{~m}^{3} . \mathrm{s}^{1}\right)$ y el control $\left(1.20 \mathrm{~m}^{3} . \mathrm{s}^{-1}\right)$ (Tabla 4).
Cuantitativamente, las curvas de tendencia expresaron que la técnica "surcos" conserva mejor el volumen de agua al final de la época de lluvias (Figura 5). De manera que la introducción de técnicas de mejoramiento de suelo en el área de estudio ha mejorado la retención de agua superficial provenientes de las precipitaciones que no se infiltra ni se evapora en forma diferenciada durante la época de lluvias, los efectos coincidieron plenamente con el estado fenológico de floración de la comunidad vegetal y el nivel de humedad de suelo de la microcuenca (Figura 5).

Los resultados demostraron que si es posible estimar la descarga de agua superficial dentro el complejo sistema del pastizal. Yan et al. (2013) observaron semejantes resultados al presente ensayo y señalaron que los principales cambios que afectaron el caudal son los cambios en las tierras de cultivo, bosques y áreas urbanas, y los cambios en los pastizales no ejercieron influencia significativa en el caudal y producción de sedimentos. En contraposición a estos resultados, Stehr et al. (2010) manifestaron que la sustitución de los pastizales con plantaciones de especies introducidas causó una reducción de la media anual de descarga en un $10 \%$. Finalmente, Nearing et al. (2011) indicaron la necesidad imperiosa de contar con un modelo para predecir la erosión del suelo de

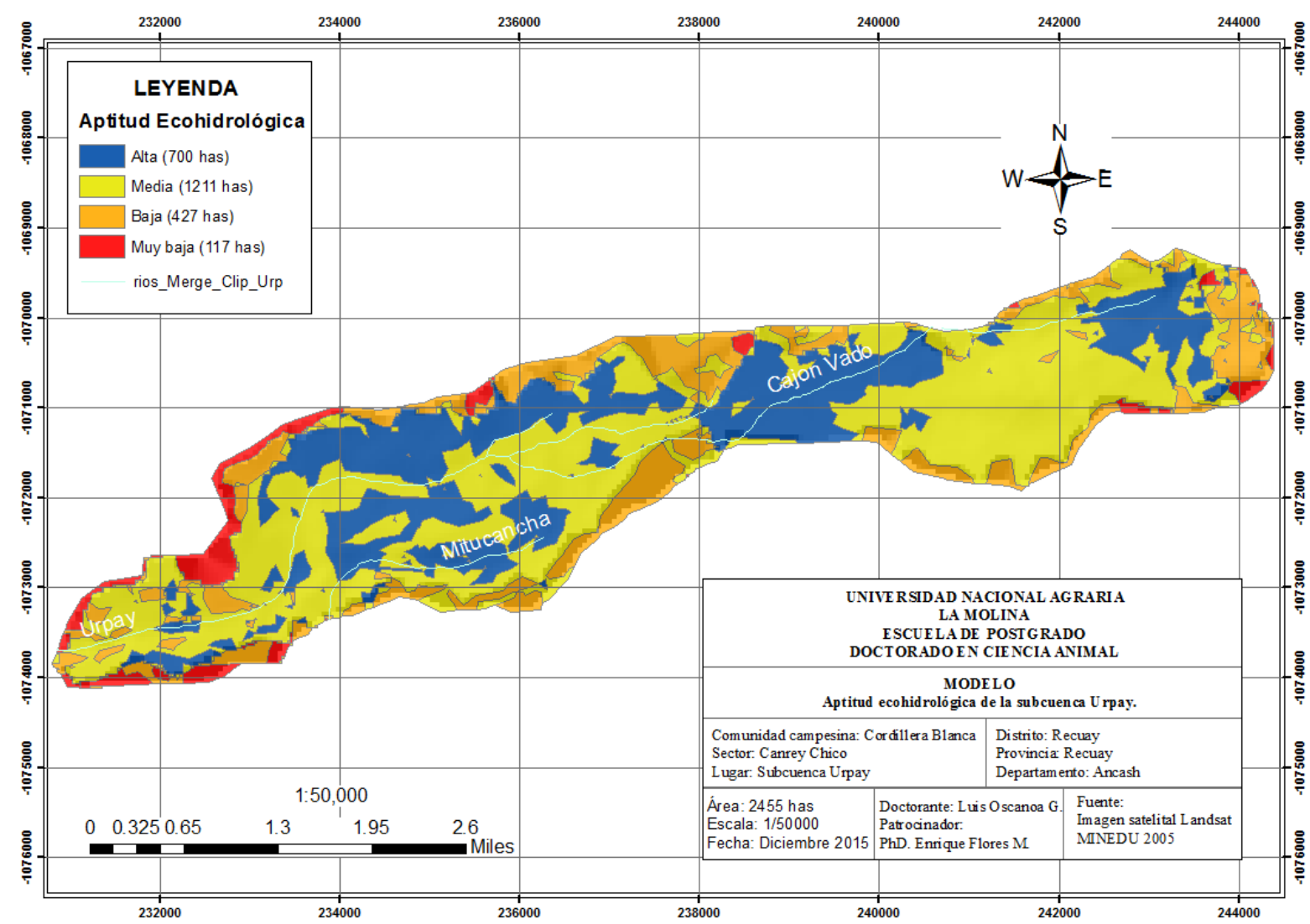

Figura 4. Modelo ecohidrológico de la subcuenca Urpay. 


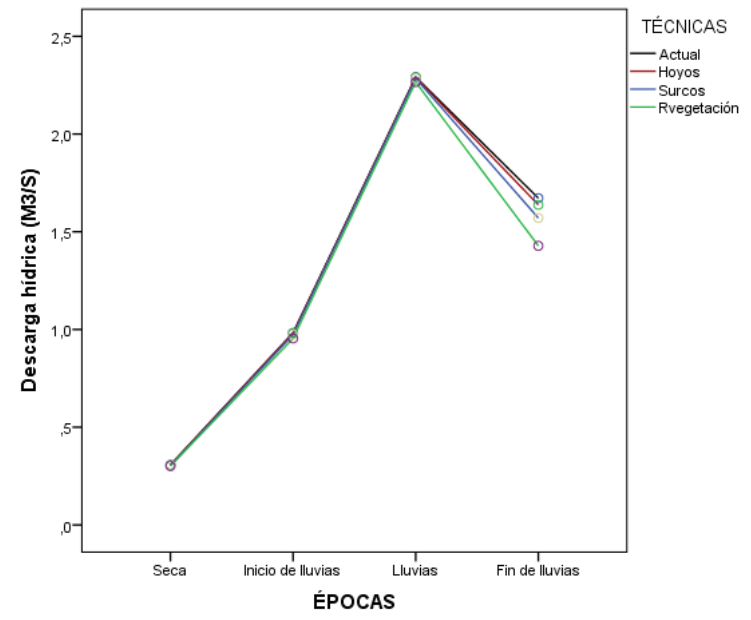

Figura 5. Medias marginales de descarga hídrica de la subcuenca Urpay.

pastoreo porque la que existen se desarrollaron para estudiar tierras de cultivo con características de procesos hidrológicos diferentes al pastizal debido a la heterogeneidad de las propiedades y uso del suelo.

\section{Conclusiones}

El estudio basado en la aplicación integrada de los principales atributos de la ciencia del pastizal (condición - C) y suelo (humedad - H) encontró que los índices son muy confiables y precisa el proceso de análisis de los cambios en la composición aérea y subsuelo de la comunidad vegetal a corto y mediano plazo. La caracterización ecohidrológica de la microcuenca Urpay logró una adecuada delimitación del territorio por aptitud y cada respuesta fue fortalecida con el análisis individualizada de los mapas temáticos de vulnerabilidad, conservación, función hidrológica y retención de agua que interactuaron en la regulación de descarga del agua al rio. En los sitios ecohidrológicos, el modelo simulado encontró mejora del rendimiento hídrico con la aplicación de técnicas de conservación de suelos ("hoyos" y "surcos") y evidenciaron que si es posible mejorar, a mínimo costo económico y de tiempo, la función ecohidrológica de los servicios ambientales, planificación participativa del territorio y diseño de programas de gestión racional de microcuencas hidrográficas basadas en el conocimiento local y científico. Las limitantes de datos de precipitación detectados por los investigadores sugieren la necesidad de desarrollar pluviómetros de tecnología intermedia, confiables y de fácil manejo por el productor.

\section{Agradecimientos}

Al Comité de Agropecuaria Local "Alli Pastu” de Canrey Chico - Huaraz, a The Mountain Institute, al Profesor Juancarlos Cruz y al Laboratorio Utilización de Pastizales de la Universidad Nacional Agraria La Molina por el apoyo de análisis de muestras; a The
Collaborative Crop Research Program/The MckNight Foundation por la beca doctoral y al Ph.D. Jorge Recharte por los apoyos y comentarios al contenido del presente artículo.

\section{Literatura citada}

Albhaisi M., Brendonck L. \& Batelaan O. 2013. Predicted impacts of land use change on groundwater recharge of the upper Berg catchment, South Africa. Water SA, 39(2): 211-220. DOI: http://dx.doi.org/10.4314/wsa.v39i2.4.

Al-seekh S.H., Mohammad A.G. \& Amor Y.A. 2009. Effect of Grazing on Soil Properties at Southern Part of West Bank Rangeland. Hebron University Research Journal. Vol.(4), No.(1), pp.(35 - 53).

Amaya G., Restrepo-Tamayo C., Vélez M.V., Vélez J.I. \& Álvarez O.D. 2009. Modelación del comportamiento hidrológico de tres cuencas en el Urabá Antioqueño Colombia. Avances en Recursos Hidraúlicos,19: 21-38. Medellín - Colombia.

Balvanshi A. \& Tiwari H.L. 2014. A Comprehensive review of runoff estimation by the curve number method. International Journal of Innovative Research in Science, Engineering and Technology, Vol. 3, Issue 11.

Blake G.R. \& Hartge K.H. 1965. Bulk Density. In: Methods of Soil Analysis, Part 1. Physical and Mineralogical Properties, Including statistics of Measurement and Sampling. Agronomy: Monograph 9: 374-390. ASASSSA.

Breshears D.D. \& Barnes F.J. 1999. Interrelationships between plant functional types and soil moisture heterogeneity for semiarid landscapes within the grassland/forest continuum: a unified conceptual model. Landscape Ecology 14: 465-478, 1999.

Deutsch E.S., Bork E.W. \& Willms W.D. 2010. Soil moisture and plant growth responses to litter and defoliation impacts in Parkland grasslands. Agriculture, Ecosystems and Environment 135: 1-9.

Di Luzio M., Srinivasan R. \& Arnold J.G. 2002. Integration of watershed tools and SWAT model into BASINS. JAWRA, 38(4): 1127-1141. DOI: https://doi.org/10.1111/j.1752-1688.2002.tb05551.x.

Eekeren N.V., Boer H., Hanegraaf M., Bokhorst J., Nierop D., Bloem J., Schouten T., Goede R. \& Brussaard L. 2010. Ecosystem services in grassland associated with biotic and abiotic soil parameters. Soil Biology \& Biochemistry, 42(9): 1491-1504. DOI: 10.1016/j.soilbio.2010.05.016.

Eviner V. 2004. Plant traits that influence ecosystem processes vary independently among species. Ecology, 85(8): 2215-2229. DOI: https://doi.org/10.1890/03$\underline{0405}$.

Flint E.L., Flint L.A., Thorne H.J. \& Boynton R. 2013. Finescale hydrologic modeling for regional landscape applications: the California Basin Characterization Model development and performance. Ecological Processes 2013.

Florez A. \& Bryant E. 1989. Manual de Pastos y Forrajes. Programa colaborativo de apoyo a la investigación en rumiantes menores. INIIA, Universidad de California. Lima - Perú.

Florez M.A., Malpartida I.E. \& San Martin F. 1991. Manual de forrajes para zonas áridas y semiáridas andinas. 
Gil R.J. 2014. Monitoreo Hidrológico en ecosistemas de la puna húmeda bajo el método de Cuencas Pareadas: C.C. Cordillera Blanca, Canrey Chico, Recuay, Ancash, Perú. Proyecto Puna-agua. Conv. Instituto de Montaña/ The MckNight Foundation. Documento en prensa.

Hagyó A., Rajkai K. \& Nagy Z. 2006. Effect of forest and grassland vegetation on soil hydrology in Mátra Mountains (Hungary). Biologia, Bratislava, 61/Suppl. 19: S261-S265.

Holdridge L.R. 1987. Ecología basada en las zonas de vida. Instituto Interamericano de Cooperación para la Agricultura (IICA). San José de Costa Rica.

Montgomery D.C. 1991. Diseño y análisis de experimentos. Arizona State University, Estados Unidos de América. Grupo Editorial Iberoamérica. Pag: 589.

Nearing M.A., Wei H., Stone J.J., Pierson F.B., Spaeth K.E., Weltz M.A., Flanagan D.C. \& Hernandez M. 2011. A rangeland hydrology and erosion model. Transactions of the ASABE, 54(3): 901-908. American Society of Agricultural and Biological Engineers. DOI: $10.13031 / 2013.37115$.

Oscanoa L. 2016. Influencia de las prácticas de conservación de suelos y mejora de la función hidrológica de praderas naturales altoandinas. Tesis para optar el grado Doctoris Philosophiae en Ciencia Animal. Universidad Nacional Agraria La Molina. Lima, Perú.

Oscanoa L. \& Flores E. 2016. Influencia de técnicas de mejora de suelos sobre la función hídrica de pastos naturales altoandinos. Ecol. Apl. Vol. $15 \mathrm{~N}^{\circ}$ 2, pp. 91-99.

Parker K.W. 1951. A method for measuring trend and range condition on National Forest Ranges. USDA. Forest Service.

Pieri L., Bittelli M., Wu Q.J., Dun S., Flanagan C.D., Rossi P.P., Ventura F. \& Salvatorelli F. 2007. Using the Water Erosion Prediction Project (WEPP) model to simulate field-observed runoff and erosion in the Apennines mountain range, Italy. Journal of Hydrology, 336: 84-97.

Pyke D.A., Herrick J.E., Shaver P. \& Pellant M. 2002. Rangeland health attributes and indicators for qualitative. J. Range Manage, 55(6): 584-597. DOI: 10.2458/azu_jrm_v55i6_pyke.

Salve R. \& Allen-Diaz B. 2001. Variations in soil moisture content in a rangeland catchment. J. Range Manage. 54(1): 44-51. DOI: $10.2307 / 4003526$.

Snymana H. \& du Preez D. 2005. Rangeland degradation in a semi-arid South Africa-II: influence on soil quality. Journal of Arid Environments, 60(3): 483-507. DOI: https://doi.org/10.1016/j.jaridenv.2004.06.005.
Stehr A., Aguayo M., Link O., Parra O., Romero F. \& Alcayaga H. 2010. Modelling the hydrologic response of a mesoscale Andean watershed to changes in land use patterns for environmental planning. Hydrol. Earth Syst. Sci., 14, 1963-1977.

Taghvaye S.E., Nohegar A., Malekian A., Hoseini M. \& Holisaz A. 2017. Estimating time of concentration in large watersheds. Paddy Water Environ 15:123-132.

Tovar O. \& Oscanoa L. 2002. Guía para la identificación de pastos naturales alto andinos de mayor importancia ganadera. Instituto de Montaña. Proyecto FOCAL Obc. Primera edición. Huaraz, Perú.

Vélez U.J. \& Botero G.A. 2011. Estimación del tiempo de concentración y tiempo de rezago en la cuenca experimental urbana de la quebrada San Luis, Manizales. Dyna, 78(165): 58-71. Medellín.

Yalew G.S., Tobias P., Schweitzer C., Liersch S., van der Kwast J., Mul M.L., van Griensven A. \& van der Zaag P. 2014. Dynamic Feedback between Land Use and Hydrology for Ecosystem Services Assessment. In: Ames D.P., Quinn N.W.T. \& Rizzoli A.E. (Eds.). Proceedings of the 7th International Congress on Environmental Modelling and Software (iEMSs), June 15-19, San Diego, California, USA. URL: https://scholarsarchive.byu.edu/iemssconference/2014/.

Yan B., Fang N.F., Zhang P.C. \& Shi Z.H. 2013. Impacts of land use change on watershed streamflow and sediment yield: An assessment using hydrologic modelling and partial least squares regression. Journal of Hydrology, 484: 26-37. DOI: https://doi.org/10.1016/j.jhydrol.2013.01.008.

Zhang L.H., Chen Y.N., Zhao R.F. \& Li W.H. 2010. Significance of temperature and soil water content on soil respiration in three desert ecosystems in Northwest China. Journal of Arid Environments, 74(10): 1200 1211.

DOI: https://doi.org/10.1016/j.jaridenv.2010.05.031.

Zhang Y., Hernandez M., Anson E., Nearing M.A., Wei M.A., Stone J.J. \& Heilman P. 2012. Modeling climate change effects on runoff and soil erosion in southeastern Arizona rangelands and implications for mitigation with conservation practices. Journal of Soil and Water Conservation, 67(5): 390-405. DOI: $10.2489 /$ jswc.67.5.390.

\footnotetext{
${ }^{1}$ Ph.D. Investigador Asociado a The Mountain Institute y Laboratorio de Ecología y Utilización de Pastizales, Departamento de Producción Animal de la Facultad de Zootecnia, Universidad Nacional Agraria La Molina, Av. La Molina s/n La Molina, Lima, Casilla Lima 12, Perú, oscanoa.luis@ gmail.com.

2 Ph.D. Profesor Principal. Laboratorio de Ecología y Utilización de Pastizales, Departamento de Producción Animal de la Facultad de Zootecnia, Universidad Nacional Agraria La Molina, Av. La Molina s/n La Molina, Lima, Casilla Lima 12, Perú, lab_pastizales@lamolina.edu.pe.
} 